\title{
Overexpression of the transmembrane protein BST-2 induces Akt and Erk phosphorylation in bladder cancer
}

\author{
YOSHINORI SHIGEMATSU ${ }^{1,2}$, NAOHIDE OUE ${ }^{1}$, YURI NISHIOKA ${ }^{1}$, NAOYA SAKAMOTO ${ }^{1}$, \\ KAZUHIRO SENTANI $^{1}$, YOHEI SEKINO ${ }^{1,2}$, SHOICHIRO MUKAI ${ }^{1}$, \\ JUN TEISHIMA $^{2}$, AKIO MATSUBARA ${ }^{2}$ and WATARU YASUI ${ }^{1}$
}

Departments of ${ }^{1}$ Molecular Pathology and ${ }^{2}$ Urology, Hiroshima University Institute of Biomedical and Health Sciences, Hiroshima 734-8551, Japan

Received May 31, 2016; Accepted March 7, 2017

DOI: $10.3892 / \mathrm{ol} .2017 .6230$

\begin{abstract}
Bladder cancer, the majority of which is urothelial carcinoma (UC), is a common malignancy worldwide. Genes encoding transmembrane/secretory proteins expressed specifically in certain cancers may be ideal biomarkers for cancer diagnosis and may represent therapeutic targets. In the present study, the expression and function of the bone marrow stromal cell antigen 2 (BST2) gene was analyzed in UC. Reverse transcription-quantitative polymerase chain reaction demonstrated that expression of BST2 in normal tissue samples was the highest in liver tissue. However, expression of BST2 in UC tissue samples was higher than in normal liver. Immunohistochemical analysis revealed weak or no staining of BST-2 in non-neoplastic mucosa, whereas UC tissue exhibited stronger and more extensive staining compared with non-neoplastic mucosa. BST-2 staining was observed mainly on UC cell membranes. In total, 28 (41\%) of 69 UC cases were positive for BST-2. UC cases positive for BST-2 were more frequently $\mathrm{T} 2 / 3 / 4$ cases [so-called muscle-invasive bladder cancer $(\mathrm{MIBC})]$ than $\mathrm{Ta} / \mathrm{is} / 1$ cases $(\mathrm{P}=0.0001)$. However, Kaplan-Meier analysis demonstrated no association between BST-2 expression and survival. BST2 small interfering RNA (siRNA)-transfected T24 cells exhibited significantly reduced cell growth relative to negative control siRNA-transfected T24 cells. The levels of phosphorylated Akt and extracellular signal-regulated kinase were lower in BST2 siRNA-transfected T24 cells than in control cells. These results suggest the involvement of BST-2 in the pathogenesis of UC. Since BST-2 is expressed on the cell membrane, BST-2 may be a good therapeutic target for MIBC.
\end{abstract}

Correspondence to: Dr Naohide Oue, Department of Molecular Pathology, Hiroshima University Institute of Biomedical and Health Sciences, 1-2-3 Kasumi, Minami-ku, Hiroshima 734-8551, Japan E-mail: naoue@hiroshima-u.ac.jp

Key words: bone marrow stromal cell antigen 2, urothelial carcinoma, bladder cancer, Akt, Erk

\section{Introduction}

Bladder cancer, the majority of which is urothelial carcinoma (UC), is a common malignancy worldwide. The prognosis of patients with UC is poor when the disease includes muscle invasion. Metastatic UC is almost uniformly fatal (1). However, progress in systemic therapies for muscle-invasive bladder carcinoma (MIBC) has been stagnant for decades, with few new systemic therapies being evaluated, until recently (2). Therefore, there is an urgent requirement for new potential therapeutic targets in UC.

Better knowledge of the changes in gene expression that occur during carcinogenesis may lead to improvements in diagnosis, treatment and prevention of cancer. Genes encoding transmembrane/secretory proteins expressed specifically in certain cancers may be ideal biomarkers for cancer diagnosis, and may represent therapeutic targets. Escherichia coli ampicillin secretion trap (CAST), a signal sequence trap method developed by Ferguson et al (3), is a unique large-scale analysis method that is useful to identify genes encoding transmembrane/secretory proteins. Using the CAST method, overexpression of the transmembrane protein bone marrow stromal cell antigen-2 (BST-2) was detected in gastrointestinal cancer (4). Furthermore, knockdown of the BST2 gene inhibits gastric cancer cell growth, suggesting that BST-2 could be a useful therapeutic target for gastric cancer (4). BST-2 is a lipid raft-associated type II transmembrane glycoprotein that is overexpressed on multiple myeloma cells $(5,6)$. Immunotherapy with a monoclonal antibody against BST-2 reduces tumor size and improves survival in a multiple myeloma mouse model (7). Such monoclonal antibody against BST-2 induces antibody-dependent cellular cytotoxicity, suggesting that it may be effective for a wide range of human malignancies (7). In addition to gastrointestinal cancer, high levels of BST-2 have been reported in neoplastic B cells (6), and in ovarian (8), breast (9), endometrial (10) and lung cancer (11). However, the expression of BST-2 has not been investigated in UC to date.

In the present study, the expression and distribution of BST-2 was examined in UC by immunohistochemistry, and potential correlations with clinicopathological factors were analyzed. In addition, the effects of BST2 knockdown on 
cell growth activity were evaluated using RNA interference (RNAi) or forced expression of BST2 in UC cell lines.

\section{Materials and methods}

Tissue samples. Using a retrospective study design, 77 primary tumors were collected from patients diagnosed with UC, who underwent surgery between April 2003 and March 2007 at Hiroshima University Hospital (Hiroshima, Japan). All patient samples were obtained with consent, and the present study was approved by the Ethical Committee for Human Genome Research of Hiroshima University (Hiroshima, Japan). All patients underwent curative resection. Only patients without preoperative radiotherapy or chemotherapy and without clinical evidence of distant metastasis were enrolled in the study. Operative mortality was defined as mortality within 30 days of patients leaving the hospital, and these patients were removed from the analysis. Postoperative follow-up was scheduled every 1, 2 or 3 months during the first 2 years after surgery, and every 6 months thereafter, unless more frequent follow-ups were deemed necessary. Chest X-ray, chest computed tomography scan and serum chemistries were performed at every follow-up visit. Follow-ups of the patients were conducted by the physician until mortality or until the date of the last documented contact. Tumor staging was performed according to the tumor-node-metastasis classification system (12).

For reverse transcription-quantitative polymerase chain reaction (RT-qPCR), $8 \mathrm{UC}$ samples were used. The samples were frozen immediately in liquid nitrogen and stored at $-80^{\circ} \mathrm{C}$ until use. A total of 14 types of normal tissue samples [namely heart (catalog no. 636532), lung (catalog no. 636524), stomach (catalog no. 636578), small intestine (catalog no. 636539), colon (catalog no. 636553), liver (catalog no. 636531), pancreas (catalog no. 636577), kidney (catalog no. 636529), bone marrow (catalog no. 636591), leukocytes (catalog no. 636592), spleen (catalog no. 636525), skeletal muscle (catalog no. 636547), brain (catalog no. 636530) and spinal cord (catalog no. 636554)] were purchased from Clontech Laboratories, Inc. (Mountainview, CA, USA).

For immunohistochemical analysis, archival formalin-fixed, paraffin-embedded tissues from 69 patients who had undergone surgical excision for UC were used. All 69 patients with UC were treated by cystectomy between April 2003 and March 2007 at the Hiroshima University Hospital (Hiroshima, Japan).

$R T$-qPCR analysis. Total RNA was extracted with an RNeasy Mini kit (Qiagen, Inc., Valencia, CA, USA), and $1 \mu \mathrm{g}$ total RNA was converted to complementary DNA (cDNA) using a First Strand cDNA Synthesis kit (GE Healthcare Life Sciences, Chalfont, UK). Quantitation of BST2 messenger RNA (mRNA) levels was performed by quantitative fluorescence detection as described previously (13). PCR was conducted using a SYBR-Green PCR Core Reagents kit (Applied Biosystems; Thermo Fisher Scientific, Inc., Waltham, MA, USA). Real-time detection of the emission intensity of SYBR Green bound to double-stranded DNA was performed with the ABI PRISM 7700 Sequence Detection System (Applied Biosystems; Thermo Fisher Scientific,
Table I. Association between BST-2 expression and clinicopathological characteristics in bladder cancer.

BST-2 expression,

$$
\mathrm{n}(\%)
$$

Characteristic

Positive Negative P-value

\begin{tabular}{lrrr}
\hline Age, years & & & \\
$\leq 70$ & $16(40)$ & $24(60)$ & 0.9083 \\
$>70$ & $12(41)$ & $17(59)$ & \\
Sex & & & \\
$\quad$ Male & $23(40)$ & $34(60)$ & 0.9327 \\
$\quad$ Female & $5(42)$ & $7(58)$ & \\
$\quad$ classification & & & \\
$\quad$ Ta/is/1 & $6(18)$ & $28(82)$ & 0.0001 \\
$\quad$ T2/3/4 & $22(63)$ & $13(37)$ & \\
Cellular atypism classification & & & \\
$\quad$ Low grade & $9(30)$ & $21(70)$ & 0.1164 \\
$\quad$ High grade & $19(49)$ & $20(51)$ & \\
Lymphatic invasion & & & \\
$\quad$ Positive & $13(46)$ & $15(54)$ & 0.8470 \\
$\quad$ Negative & $13(35)$ & $24(65)$ & \\
Vascular invasion & & & \\
$\quad$ Positive & $9(60)$ & $6(40)$ & 0.0714 \\
$\quad$ Negative & $17(34)$ & $33(66)$ & \\
\hline
\end{tabular}

BST-2, bone marrow stromal cell antigen-2.

Inc.). $\beta$-Actin-specific PCR products were amplified from the same RNA samples and served as an internal control. Quantitation of ACTB mRNA levels was performed by quantitative fluorescence detection as described previously (13). RT-qPCR reactions were performed in triplicate for each sample primer set, and the mean of the three experiments was used as the relative quantification value (14). Primer sequences for $B S T 2$ were forward, 5'-CAG AAG GGC TTT CAG GAT GT-3' and reverse, 5'-TTC TCA GTC GCT CCA CCT CT-3'. Primer sequences for $A C T B$ were forward, 5'-TCA CCG AGC GCG GCT-3' and reverse, 5'-TAA TGT CAC GCA CGA TTT CCC-3'. The thermocycling conditions were used as described previously (13).

Immunohistochemistry. Immunohistochemical analysis was performed with the EnVison+ Rabbit Peroxidase Detection System (Dako; Agilent Technologies GmbH, Waldbronn, Germany) as described previously (15). As the primary antibody, a rabbit polyclonal anti-BST-2 antibody was used (dilution, 1:50; catalog no. HPA017060, Sigma-Aldrich; Merck KGaA, Darmstadt, Germany) (4). A result was considered positive if $\geq 10 \%$ of cancer cells were stained. When $<10 \%$ of cancer cells were stained, the immunostaining was considered negative.

Cell lines. Two cell lines derived from human UC (T24 and $\mathrm{KMBC} 2$ ) were used. Both cell lines were purchased from 


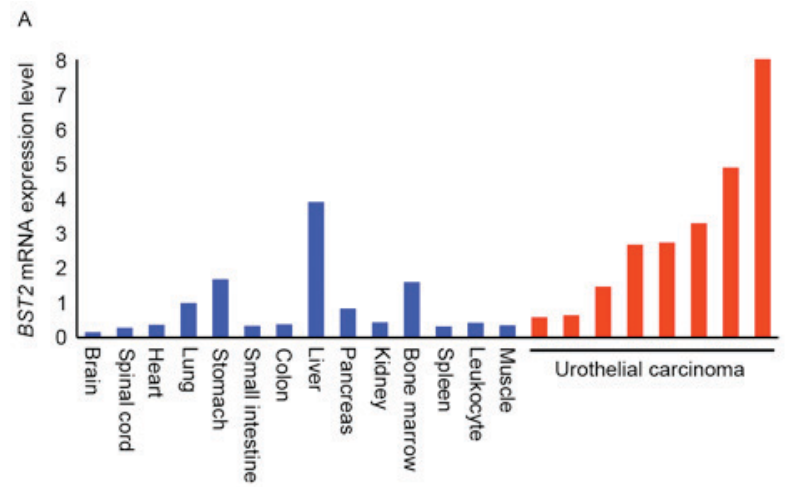

B

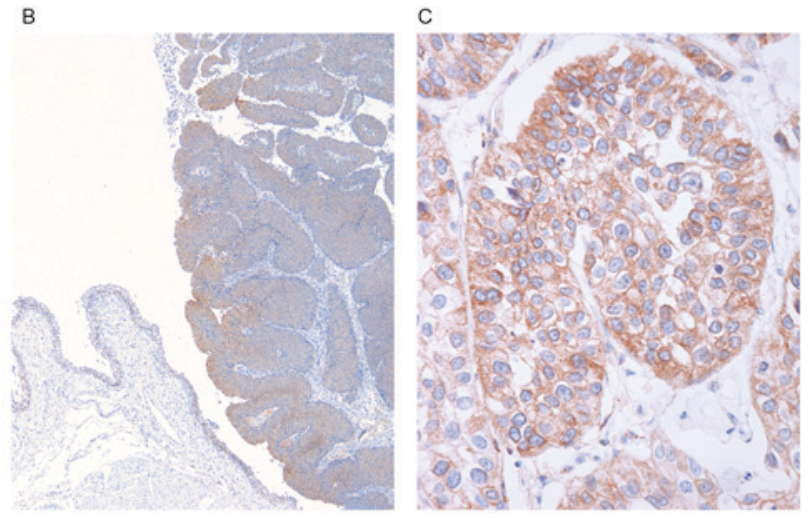

Figure 1. Expression of BST2 in UC. (A) RT-qPCR analysis of BST2 expression in 14 types of normal tissue and $8 \mathrm{UC}$ samples. RT-qPCR reactions were performed in triplicate for each sample, and the mean of three experiments was used as the relative quantification value. (B) Immunohistochemical analysis of BST-2 in non-neoplastic mucosa and UC. Original magnification, $\mathrm{x} 100$. (C) Immunostaining of BST-2 in UC. BST-2 staining was observed on the cell membranes of UC cells. Original magnification, $x 400$. UC, urothelia carcinoma; BST2, bone marrow stromal cell antigen 2; mRNA, messenge RNA; RT-qPCR, reverse transcription quantitative polymerase chain reaction.

the Japanese Collection of Research Bioresources Cell Bank (Osaka, Japan), and were maintained in RPMI 1640 medium (Nissui Pharmaceutical Co., Ltd., Tokyo, Japan) containing $10 \%$ fetal bovine serum (BioWhittaker; Lonza, Basel, Switzerland) at $37^{\circ} \mathrm{C}$ in a humidified atmosphere with $5 \% \mathrm{CO}_{2}$.

RNAi, expression vector and cell growth assay. Small interfering RNA (siRNA) oligonucleotides targeting BST2 and a negative control siRNA were purchased from Invitrogen (Thermo Fisher Scientific, Inc.). Two independent BST2 siRNA oligonucleotide sequences were used (catalog nos. 251993co3 and 251993co5). Transfection was performed using Lipofectamine RNAiMAX (Invitrogen; Thermo Fisher Scientific, Inc.) as described previously (16). Briefly, 60 pmol siRNA and $10 \mu$ l Lipofectamine RNAiMAX were mixed in $1 \mathrm{ml}$ RPMI 1640 medium (10 nmol/1 final concentration). After $20 \mathrm{~min}$ of incubation, the mixture was added to the cells $(100,000$ cells $/ \mathrm{ml})$, and then the cells were plated in culture dishes. At $48 \mathrm{~h}$ post-transfection, the cells were analyzed.

For constitutive expression of the BST2 gene, cDNA was amplified by PCR and then subcloned into the pcDNA3.1 vector (Invitrogen; Thermo Fisher Scientific, Inc.). The resultant pcDNA-BST2 expression vector was transfected into KMBC2 cells with FuGENE6 (Roche Diagnostics, Basel, Switzerland) according to the manufacturer's protocol. KMBC2 cells were selected as the cell line express low levels of BST-2.

To examine cell growth, MTT assays were performed. The cells were seeded at a density of 2,000 cells per well in 96-well plates. Cell growth was examined after 1, 2 and 4 days. Three independent experiments were performed. The mean and standard error were calculated for each experiment.

Western blot analysis. Cells were lysed as described previously (17). The lysates (40 $\mu \mathrm{g}$ protein) were solubilized in Laemmli sample buffer (catalog no. S3401, Sigma-Aldrich; Merck KGaA) by boiling and then subjected to $10 \%$ SDS-PAGE, followed by electrotransfer onto a nitrocellulose membrane. An anti-BST-2 monoclonal antibody (catalog no. H00000684-B02P) was purchased from Abnova (Taipei, Taiwan) (4). Peroxidase-conjugated anti-mouse immunoglobulin $\mathrm{G}$ was used as the secondary antibody (4). Immunocomplexes were visualized with the Amersham ECL Western Blotting Detection kit (GE Healthcare Life Sciences). $\beta$-Actin (catalog no. A5441; Sigma-Aldrich; Merck KGaA) was also stained as a loading control (4).

Statistical methods. Associations between clinicopathological parameters and BST-2 expression were analyzed by the Fisher's exact test. Kaplan-Meier survival curves were constructed for BST-2-positive and -negative patients. Survival rates were compared between BST-2-positive and -negative groups. Differences between survival curves were evaluated for statistical significance by the log-rank test. $\mathrm{P}<0.05$ was considered to indicate a statistically significant difference. SPSS version 8.0 software was used for these analyses (SPSS Inc., Chicago, IL, USA).

\section{Results}

Expression of BST2 in UC. RT-qPCR analysis of BST2 was first performed in 14 types of normal tissue samples and 8 UC tissue samples (Fig. 1A). All 8 UC tissue samples were randomly selected. Among the various normal tissue samples, abundant BST2 mRNA expression was detected in normal lung, stomach, liver and bone marrow samples. The expression of BST2 in these normal tissue samples was highest in the liver. However, expression of BST2 in UC tissue samples was higher than in normal liver.

Next, immunohistochemistry was performed on 69 UC tissue samples. In non-neoplastic mucosa, weak or no staining of BST-2 was observed in urothelial and stromal cells, whereas UC tissue exhibited stronger and more extensive staining compared with non-neoplastic mucosa (Fig. 1B). BST-2 staining was observed mainly on UC cell membranes (Fig. 1C). Numerous UC cases displayed heterogeneity of BST-2 staining, and the percentage of BST-2-stained UC cells ranged from 0 to $70 \%$. A tendency for upregulation of BST-2 was not observed at the invasive front. In total, 28 (41\%) of $69 \mathrm{UC}$ cases were positive for BST-2 expression.

Next, the association of BST-2 staining with clinicopathological characteristics was examined (Table I). UC cases positive for BST-2 were more frequently T2/3/4 cases (so-called MIBC) than $\mathrm{Ta} / \mathrm{is} / 1$ cases $(\mathrm{P}=0.0001)$. However, 


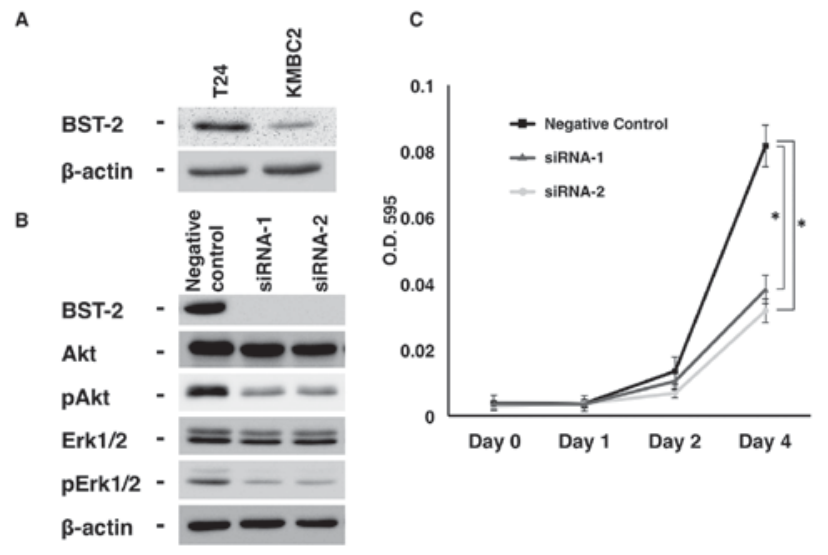

Figure 2. Effect of $B S T 2$ inhibition in T24 urothelial carcinoma cells. (A) Western blot analysis of BST-2, Akt, pAkt, Erk1/2 and pErk1/2 in lysates of T24 cells transfected with BST2 siRNA or negative control siRNA. $\beta$-Actin was used as a loading control. (B) Effect of BST2 knockdown on the growth of T24 cells. Cell viability was assessed by MTT assays at days 1,2 and 4 after seeding on 96 -well plates. Bars and error bars indicate the mean and standard error, respectively, of three independent experiments. siRNA, small interfering RNA; p, phosphorylated; Erk, extracellular signal-regulated kinase; $B S T 2$, bone marrow stromal cell antigen 2; O.D., optical density. ${ }^{*} \mathrm{P}<0.001$.

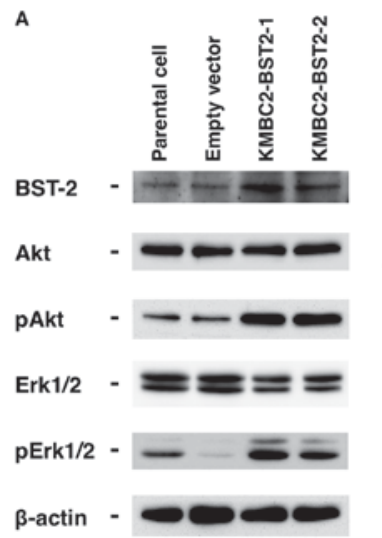

B

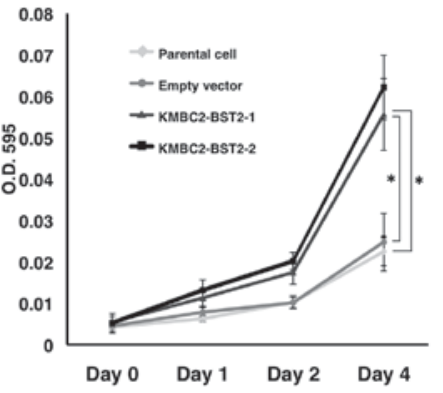

Figure 3. Effect of $B S T 2$ overexpression in KMBC2 urothelial carcinoma cells. (A) Western blot analysis of BST-2, Akt, pAkt, Erk1/2 and pErk1/2 in lysates of BST2-transfected and empty vector-transfected $\mathrm{KMBC} 2$ cells. $\beta$-Actin was used as a loading control. (B) Effect of BST-2 on the growth of KMBC 2 cells. Cell viability was assessed by MTT assays at days 1,2 and 4 after seeding on 96 -well plates. Bars and error bars indicate the mean and standard error, respectively, of three independent experiments. Erk, extracellular signal-regulated kinase; $\mathrm{p}$, phosphorylated; $B S T 2$, bone marrow stromal cell antigen 2; O.D., optical density. ${ }^{*} \mathrm{P}<0.001$.

Kaplan-Meier analysis demonstrated no association between BST-2 expression and survival $(\mathrm{P}=0.4602)$ (data not shown).

Effect of BST2 inhibition on cell growth. Previously, it was demonstrated that inhibition of $B S T 2$ by siRNA reduces cell growth, and that the levels of phosphorylated epidermal growth factor receptor (EGFR), Akt and extracellular signal-regulated kinase (Erk) are lower in BST2 siRNA-transfected gastric cancer cells than in control cells (4). However, the biological function of BST-2 has not been investigated in UC cells thus far. Therefore, the present study examined the effect of $B S T 2$ inhibition on cell growth using two different siRNA sequences (catalog nos. 251993co3 and 251993co5; Invitrogen; Thermo Fisher Scientific, Inc.). T24 UC cells were selected, which express detectable levels of BST-2 protein (Fig. 2A). BST-2 expression was substantially suppressed by treatment with siRNA1 and siRNA2, as shown by western blotting (Fig. 2B). Next, cell growth was analyzed by MTT assays, which revealed that BST2 siRNA1- and siRNA2-transfected T24 cells exhibited significantly reduced cell growth relative to negative control siRNA-transfected T24 cells (Fig. 2B).

Since EGFR activates the Ras-mitogen-activated protein kinase kinase-Erk and Akt-phosphatidylinositol4,5-bisphosphate 3-kinase signaling pathways, thus leading to cancer cell proliferation and survival (18), the effect of $B S T 2$ inhibition on EGFR signaling was analyzed in the present study. The results indicated that the levels of phosphorylated Akt and Erk were lower in BST2 siRNA1- and siRNA2-transfected T24 cells than in control cells (Fig. 2A).

Effect of forced expression of BST2 on cell growth. To further investigate the biological significance of BST-2, the KMBC2 UC cell line was stably transfected with a vector expressing $B S T 2$. KMBC2 cells were selected due to their low expression levels of BST-2 compared with T24 cells (Fig. 2A). Clones were selected with G418 and examined for BST-2 expression by western blotting. Two clones, KMBC2-BST2-1 and KMBC2-BST2-2, expressed BST-2 protein at significantly higher levels than KMBC2 cells transfected with the empty vector (Fig. 3A). To determine the effect of BST-2 on cell growth, MTT assays were performed. BST2-transfected KMBC2 cells exhibited significantly increased cell growth relative to empty vector-transfected KMBC2 cells (Fig. 3B). The effect of $B S T 2$ overexpression on EGFR signaling was also analyzed. The levels of phosphorylated Akt and Erk were higher in BST2-transfected KMBC2 cells compared with empty vector-transfected $\mathrm{KMBC} 2$ cells (Fig. 3A).

\section{Discussion}

Platinum-based chemotherapy is currently the standard treatment in previously untreated patients with metastatic $\mathrm{UC}$ and is associated with a median overall survival of 15 months (19). The prognosis for patients who relapse following platinum-based chemotherapy is poor, with median survival times ranging from 5 to 7 months, and no known life-prolonging treatments are available (20). Therefore, a novel therapeutic target is required for UC. In the present study, the expression of BST-2, a lipid raft-associated type II transmembrane glycoprotein, was analyzed in UC. Among the various normal tissue samples examined, expression of $B S T 2$ was the highest in the liver. However, expression of $B S T 2$ in UC tissue samples was higher than in normal liver, suggesting that BST-2 is a therapeutic target with fewer adverse effects compared with other anticancer drugs for various cancer types, including UC. Immunohistochemical analysis revealed BST-2 expression on the cell membrane, and $41 \%$ of UC cases were positive for BST-2. UC cases positive for BST-2 were more frequently $\mathrm{T} 2 / 3 / 4$ cases than 
$\mathrm{Ta} /$ is/ 1 cases. Taken together, these results suggest that BST-2 serves an important role in the pathogenesis of UC and is a good therapeutic target for T2/3/4 UC (also known as MIBC) cases.

Previously, our group reported that $36 \%$ of gastric cancer cases, $46 \%$ of colorectal cancer cases and $27 \%$ of esophageal cancer cases were positive for BST-2 (4). Furthermore, high levels of BST-2 have been reported in ovarian cancer (8), neoplastic B cells (6), breast cancer (9), endometrial cancer (10) and lung cancer (11), indicating that BST-2 expression is a common event in human malignancies. Ozaki et al (7) reported that a monoclonal antibody against BST-2 induces antibody-dependent cellular cytotoxicity, and that immunotherapy using this anti-BST-2 antibody reduces tumor size and improves survival in a multiple myeloma mouse model. Therefore, immunotherapy using a similar anti-BST-2 antibody may also improve the survival of UC patients.

In the present study, BST2 siRNA-transfected T24 UC cells exhibited significantly reduced cell growth relative to negative control siRNA-transfected T24 UC cells in MTT assays. Furthermore, BST2-transfected KMBC2 UC cells displayed significantly increased cell growth relative to empty vector-transfected KMBC2 UC cells in MTT assays. The levels of phosphorylated Akt and Erk were lower in BST2 siRNA-transfected T24 UC cells than in control cells. It was also demonstrated that the levels of phosphorylated Akt and Erk were higher in BST2-transfected KMBC2 UC cells than in empty vector-transfected KMBC2 UC cells. Since the phosphorylation of Akt and Erk inhibits apoptosis (21), these results suggest that apoptosis could be induced in BST2-transfected KMBC2 UC cells. EGFR overexpression in UC is correlated with high tumor grade, muscle invasiveness, tumor recurrence and overall survival (22). Although the underlying mechanisms remain unclear, it is possible that a monoclonal antibody against BST-2 could inhibit EGFR signaling and induce apoptosis in UC cells. The safety and efficacy of a humanized anti-BST-2 antibody has been investigated in a phase I/II clinical study on patients with relapsed or refractory multiple myeloma and has shown mild and manageable side effects (23). Thus, the efficacy of this humanized anti-BST-2 antibody should be examined in a clinical study on patients with metastatic UC or those who relapse following platinum-based chemotherapy.

In summary, the present study demonstrated overexpression of BST-2 in UC, particularly in T2/3/4 UC (MIBC) cases. Since a humanized anti-BST-2 antibody is currently available, the efficacy of such antibody should be examined in a clinical study. Although lower levels of phosphorylated Akt and Erk were detected in BST2 siRNA-transfected UC cells than in control cells, the underlying mechanisms remain unclear. Identification of BST-2 signaling will further improve the understanding of the basic biology of BST-2.

\section{Acknowledgements}

The authors thank Mr. Shinichi Norimura (Hiroshima University, Hiroshima, Japan) for his excellent technical assistance and advice. The present study was supported by Grants-in-Aid for Scientific Research (B-15H04713) and Challenging Exploratory
Research (grant nos. 26670175 and 16K15247) from the Japan Society for the Promotion of Science. The present study was also supported by the Takeda Science Foundation.

\section{References}

1. Sylvester RJ, van der Meijden AP, Oosterlinck W, Witjes JA, Bouffioux C, Denis L, Newling DW and Kurth K: Predicting recurrence and progression in individual patients with stage Ta T1 bladder cancer using EORTC risk tables: A combined analysis of 2596 patients from seven EORTC trials. Eur Urol 49: 466-477, 2006.

2. Knollman H, Godwin JL, Jain R, Wong YN, Plimack ER and Geynisman DM: Muscle-invasive urothelial bladder cancer: An update on systemic therapy. Ther Adv Urol 7: 312-330, 2015

3. Ferguson DA, Muenster MR, Zang Q, Spencer JA, Schageman JJ, Lian Y, Garner HR, Gaynor RB, Huff JW, Pertsemlidis A, et al: Selective identification of secreted and transmembrane breast cancer markers using Escherichia coli ampicillin secretion trap. Cancer Res 65: 8209-8217, 2005.

4. Mukai S, Oue N, Oshima T, Mukai R, Tatsumoto Y, Sakamoto N, Sentani K, Tanabe K, Egi H, Hinoi T, et al: Overexpression of Transmembrane Protein BST2 is Associated with Poor Survival of Patients with Esophageal, Gastric, or Colorectal Cancer. Ann Surg Oncol 24: 594-602, 2017.

5. Kupzig S, Korolchuk V, Rollason R, Sugden A, Wilde A and Banting G: Bst-2/HM1.24 is a raft-associated apical membrane protein with an unusual topology. Traffic 4: 694-709, 2003.

6. Goto T, Kennel SJ, Abe M, Takishita M, Kosaka M, Solomon A and Saito S: A novel membrane antigen selectively expressed on terminally differentiated human B cells. Blood 84: 1922-1930, 1994.

7. Ozaki S, Kosaka M, Wakatsuki S, Abe M, Koishihara Y and Matsumoto T: Immunotherapy of multiple myeloma with a monoclonal antibody directed against a plasma cell-specific antigen, HM1.24. Blood 90: 3179-3186, 1997.

8. Walter-Yohrling J, Cao X, Callahan M, Weber W, Morgenbesser S, Madden SL, Wang C and Teicher BA: Identification of genes expressed in malignant cells that promote invasion. Cancer Res 63: 8939-8947, 2003.

9. Cai D, Cao J, Li Z, Zheng X, Yao Y, Li W and Yuan Z: Up-regulation of bone marrow stromal protein 2 (BST2) in breast cancer with bone metastasis. BMC Cancer 9: 102, 2009.

10. Yokoyama T, Enomoto T, Serada S, Morimoto A, Matsuzaki S, Ueda Y, Yoshino K, Fujita M, Kyo S, Iwahori K, et al: Plasma membrane proteomics identifies bone marrow stromal antigen 2 as a potential therapeutic target in endometrial cancer. Int J Cancer 132: 472-484, 2013.

11. Wang W, Nishioka Y, Ozaki S, Jalili A, Abe S, Kakiuchi S, Kishuku M, Minakuchi K, Matsumoto T and Sone S: HM1.24 (CD317) is a novel target against lung cancer for immunotherapy using anti-HM1.24 antibody. Cancer Immunol Immunother 58: 967-976, 2009.

12. Sobin LH, Gospodarowicz MK and Wittekind CH (eds): TNM classification of malignant tumours. 7th edition. New York: Wiley-Liss, pp262-265, 2009.

13. Kondo T, Oue N, Yoshida K, Mitani Y, Naka K, Nakayama H and Yasui W: Expression of POT1 is associated with tumor stage and telomere length in gastric carcinoma. Cancer Res 64: 523-529, 2004.

14. Livak KJ and Schmittgen TD: Analysis of relative gene expression data using real-time quantitative PCR and the 2(-Delta Delta C(T)) Method. Methods 25: 402-408, 2001.

15. Oue N, Naito Y, Hayashi T, Takigahira M, Kawano-Nagatsuma A, Sentani K, Sakamoto N, Zarni Oo H, Uraoka N, Yanagihara K, et al: Signal peptidase complex 18, encoded by SEC11A, contributes to progression via TGF- $\alpha$ secretion in gastric cancer. Oncogene 33: 3918-3926, 2014.

16. Sakamoto N, Oue N, Sentani K, Anami K, Uraoka N, Naito Y, Oo HZ, Hinoi T, Ohdan H, Yanagihara K, et al: Liver-intestine cadherin induction by epidermal growth factor receptor is associated with intestinal differentiation of gastric cancer. Cancer Sci 103: 1744-1750, 2012.

17. Yasui W, Ayhan A, Kitadai Y, Nishimura K, Yokozaki H, Ito $\mathrm{H}$ and Tahara E: Increased expression of p34cdc2 and its kinase activity in human gastric and colonic carcinomas. Int J Cancer 53: 36-41, 1993. 
18. Li S, Schmitz KR, Jeffrey PD, Wiltzius JJ, Kussie P and Ferguson KM: Structural basis for inhibition of the epidermal growth factor receptor by cetuximab. Cancer Cell 7: 301-311, 2005.

19. von der Maase H, Sengelov L, Roberts JT, Ricci S, Dogliotti L, Oliver T, Moore MJ, Zimmermann A and Arning M: Long-term survival results of a randomized trial comparing gemcitabine plus cisplatin, with methotrexate, vinblastine, doxorubicin, plus cisplatin in patients with bladder cancer. J Clin Oncol 23: 4602-4608, 2005

20. Rosenberg JE, Hoffman-Censits J, Powles T, van der Heijden MS Balar AV, Necchi A, Dawson N, O'Donnell PH, Balmanoukian A, Loriot Y, et al: Atezolizumab in patients with locally advanced and metastatic urothelial carcinoma who have progressed following treatment with platinum-based chemotherapy: A single-arm, multicentre, phase 2 trial. Lancet 387: 1909-1920, 2016.
21. Takeuchi K and Ito F: EGF receptor in relation to tumor development: Molecular basis of responsiveness of cancer cells to EGFR-targeting tyrosine kinase inhibitors. FEBS J 277: 316-326, 2010.

22. Choudhury NJ, Campanile A, Antic T, Yap KL, Fitzpatrick CA, Wade JL III, Karrison T, Stadler WM, Nakamura Y and O'Donnell PH: Afatinib activity in platinum-refractory metastatic urothelial carcinoma in patients With ERBB alterations. J Clin Oncol 34: 2165-2171, 2016.

23. Harada T and Ozaki S: Targeted therapy for HM1.24 (CD317) on multiple myeloma cells. Biomed Res Int 2014: 965384, 2014. 